\title{
Remoção de atrazina e metabólitos pela filtração lenta com leito de areia e carvão ativado granular
}

\author{
Removal of atrazine and metabolites through slow filtration by sand and \\ granular activated carbon
}

\author{
Edumar Ramos Cabral Coelho \\ Engenheira Civil. Doutora em Hidráulica e Saneamento. Professora Associada do Departamento de Engenharia Ambiental da Universidade Federal do \\ Espírito Santo (UFES) - Vitória (ES), Brasil.
}

Luis Di Bernardo

Engenheiro Civil. Doutor em Hidráulica e Saneamento. Professor Colaborador da Universidade de Ribeirão Preto (UNAERP) - Ribeirão Preto (SP), Brasil.

\section{Resumo}

A atrazina (ATZ)é um herbicida largamente utilizado no mundo, sendo encontrada associada aos seus produtos de degradação em águas superficiais e subterrâneas. Pertence à classe das s-triazinas e, juntamente com os metabólitos clorados deetilatrazina (DEA) e deisopropilatrazina (DIA), possui potencial carcinogênico e toxicidade como disruptores endócrinos. A limitação dos processos que empregam a coagulação química na remoção de ATZ, a conhecida capacidade do carvão ativado em remover microcontaminantes em água e o risco que a ATZ e seus metabólitos apresentam à saúde motivaram o estudo da filtração lenta com leito de areia e carvão ativado granular. Os resultados apontaram a eficiência do processo de filtração lenta com camada intermediária de carvão ativado granular na remoção de ATZ e a limitação deste na remoção dos metabólitos DEA, DIA e deetilhidroxiatrazina (DEHA).

Palavras-chave: atrazina; filtração lenta; carvão ativado granular; metabólitos.

\begin{abstract}
Atrazine (ATZ) is widely used as herbicide, commonly found in association to its degradation products in surface water and groundwater. It belongs to the class of s-triazines and together with the chlorinated metabolites dieethylatrazine (DEA) and deisopropilatrazine (DIA) have carcinogenic potential and toxicity as endocrine disruptors. The limitation of the processes employing chemical coagulation in the removal of atrazine, the known ability of activated carbon to remove microcontaminants in water and the risk that atrazine and the potential toxicity to human health of its metabolits motivated the study of slow sand filtration bed combined with granular activated carbon. The results showed the high efficiency of the slow filtration process with intermediate layer of granular activated carbon in the removal of atrazine and its limitation on the removal of the metabolites DEA, DIA and diethylhidroxiatrazine (DEHA).
\end{abstract}

Keywords: atrazine; slow sand filtration; granular activated carbon; metabolites.

\section{Introdução}

\section{Atrazina}

A atrazina (ATZ) é caracterizada pela sua abrangente aplicação, alta persistência em diferentes ambientes aquáticos e capacidade de produzir efeitos nos sistemas neuroendócrino e reprodutivo, além de ser classificada como de potencial carcinogênico (BIRADAR e RAYBURN, 1995; FRIEDMANN, 2002; GIBSON e KOIFMAN, 2008; USEPA, 2009). Como disruptor endócrino, pode interferir na fe-

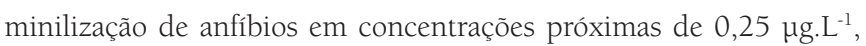
segundo Hayes et al. (2006), e pode também influenciar o sistema reprodutivo humano, induzindo a redução na contagem de espermatozoides (SWAN et al., 2003).

Análises sistemáticas de micropoluentestêm mostrado a característica sazonal de várias espécies entre a ocorrência e não ocorrência no meio ambiente. Nos Estados Unidos e Europa tem sido encontrada ATZ em mananciais em concentração variando de 2,9 a $89 \mu \mathrm{g} . \mathrm{L}^{-1}$ (CEREJEIRA et al., 2003; MILTNER et al., 1989; UETA et al., 2001). Segundo Graymore et al. (2001) a contaminação dos mananciais pode atingir concentração para ATZ tão altas quanto $1.000 \mu \mathrm{g} . \mathrm{L}^{-1}$ após as primeiras chuvas.

No meioambiente as três principais vias de degradação da ATZ são: dealquilação biológica, hidrólise química e hidrólise biológica. A hidrólise da ligação carbono-cloro induz a formação de 
hidroxiatrazina, entre eles a dietilhidroxiatrazina (DEHA) que é um dos principais produtos de degradação no solo e na água. De acordo com Graymore et al. (2001), os metabólitos mais comuns são deetilatrazina (DEA), deisopropilatrazina (DIA), DEHA, hidroxiatrazina (HA) e didealkilatrazina (DDA). ATZ é moderadamente hidrofílica e seus metabólitos apresentam maior solubilidade podendo indicar baixa remoção pelo processo de adsorção (ADAMS e WATSON, 1996). Em águas superficiais, as concentrações dos metabólitos clorados são geralmente menores que a ATZ (WHO, 2010). No Quadro 1 estão apresentadas a estrutura química e a solubilidade dos compostos estudados nesta pesquisa, ATZ, DEA, DIA e DEHA.

Pesquisas têm apontado que os produtos cloradosde degradaçãoda ATZ, DEA, DIA e DDA causam efeito tóxico no sistema neuroendócrino. Apesar dessas evidências existem escassos dados referentes àpresença destes no meio ambiente e seus efeitos na saúde (JIANG e ADAMS, 2006; HAYES et al., 2006; OH et al., 2003).

No Brasil, a ATZ é utilizada no controle de ervas daninhas e nas culturasde milho, soja e cana-de-açúcar. Apesar de poucos estudos de monitoramento, a ATZ vem sendo encontrada em alguns mananciais (BORTOLUZZI et al., 2006; LAABS et al., 2002). A Portaria $n^{\circ}$ 2.914/2011 do Ministério da Saúde estabelece para a ATZ valor máximo permitido de $2 \mu \mathrm{g}$. $\mathrm{L}^{-1}$ não fazendo referência aos metabólitos (BRASIL, 2011).A legislação europeia é mais restritiva e o EC Drinking Water Directive inclui um limite individual para agrotóxicos de 0,1e 0,5 $\mu \mathrm{g} . \mathrm{L}^{-1}$ para somatória dos agrotóxicos presentes na amostra. Devido a evidência da presença da ATZ e seus metabólitos em mananciais a Organização Mundial de Saúde (WHO, 2010) elaborou um estudo com a intenção de incluir os metabólitos DEA, DIA, diaminoclorotriazina (DACT) e DEHA em seu padrão de potabilidade.
Os processos de tratamento de água como coagulação química, filtração rápida e filtração lenta em areia podem reduzir a concentração de agrotóxicos com baixa solubilidade, mas nem sempre estes valores enquadram-se dentro dos padrões de potabilidade (THUY et al., 2008). Foster et al. (1991) estudaram a remoção de ATZ pelos processos de coagulação química e filtração lenta em água do rio Tâmisa na Inglaterra. Para concentração média de ATZ na água do rio igual a $0,3 \mu \mathrm{g} \cdot \mathrm{L}^{-1}$ os efluentes dos dois tratamentos apresentaram concentração média de $0,22 \mu \mathrm{g} . \mathrm{L}^{-1}$, não atendendo o padrão de potabilidade europeu que é de 0,1 $\mu \mathrm{g} \cdot \mathrm{L}^{-1}$. Jiang e Adams (2006) estudaram, além da ATZ, a remoção de simazina (SIM), propazina (PROP) e os metabólitos DEA, DIA e DDA, todos com concentração inicial de $3 \mu \mathrm{g} . \mathrm{L}^{-1}$, nos processos de coagulação-floculação-sedimentação com sais de alumínio e ferro seguido de cloração e encontraram que os compostos cloro-s-triazínicos não foram removidos. Por outro lado autilização de carvão ativado em pó mostrou-se como tecnologia indicada para remoção das cloro-s-triazinas com dosagens superiores a $10 \mathrm{mg} . \mathrm{L}^{-1}$.

\section{Filtração lenta associada a carvão ativado granular}

Apesar de sua simplicidade operacional, a filtração lenta é um processo apropriado para remoção de matéria orgânica e inorgânica em suspensão e organismos patogênicos, sendo o principal mecanismo de remoção o processo biológico resultante da formação de uma comunidade biológica ao redor dos grãos do leito. Em 1980, no Reino Unido, a filtração lenta era o único processo utilizado em cerca de $27,6 \%$ do total de água tratada, e, além disso, em algumas regiões da Inglaterra mais de $70 \%$ da água tratada estava envolvida com a filtração lenta como processo secundário de tratamento (MBWETTE;

Quadro 1 - Estrutura química e solubilidade dos compostos atrazina, dietilatrazina, deisopropilatrazina e dietilhidroxiatrazina. 
GRAHAM, 1990). A filtração lenta tem sido utilizada para produção de água potável em comunidades rurais há muitas décadas e em países em desenvolvimento, tais como, Colômbia, China e Índia. Em países desenvolvidos, Holanda, Inglaterra e Estados Unidos, tem sido aplicada como tecnologia para reduzir contaminantes específicos após o tratamento convencional (PROSAB, 1999).

Embora a filtração lenta seja um processo capaz de melhorar a qualidade física, química e bacteriológica de uma água, a sua utilização, como na maioria dos processos de tratamento, é limitada pela qualidade da água bruta afluente ao filtro e sua utilização fica restrita a águas que apresentam valores de cor verdadeira, turbidez e teor de sólidos suspensos relativamente baixos (PROSAB, 1999). Outras limitações da filtração lenta que podem ser apontadas são: baixa remoção de minerais complexados, matéria orgânica (substâncias húmicas) e micropoluentes (BAUER et al., 1996). Para a remoção de alguns agrotóxicos, como mercoprop e 4-Cloro-2methyl-phenoxyacetic acid (MCPA), a filtração lenta tem se mostrado efetiva, chegando atingir a remoção de $100 \%$, para concentrações iniciais de 1,0 $\mu \mathrm{g} . \mathrm{L}^{-1}$ sendo que a mesma eficiência não foi observada com relação à ATZ (FOSTER et al., 1991). Woudneh et al. (1996) estudaram a remoção de ATZ em um sistema de pré-filtração de fluxo ascendente em pedregulho com três unidades seguidas de filtro lento de areia, utilizando água do rio Tillingbourne, na Inglaterra. A ATZ foi adicionada ao afluente dos pré-filtros em concentração de 3,1 a 5,0 $\mu \mathrm{g} . \mathrm{L}^{-1}$ por 33 dias, e o tempo de contato variou de 1,6 a 6 horas e a taxa de filtração entre 1,7 a $3,8 \mathrm{~m}^{3} \cdot \mathrm{m}^{-2} \cdot \mathrm{dia}^{-1}$. Segundo os autores não ocorreu diminuição no conteúdo da ATZ devido ao baixo coeficiente cinético de degradação e toxicidade aos microrganismos.

Uma revisão dos limites de agrotóxico no EC Drinking Water Directive em 1980 conduziu os pesquisadores, na Europa, a buscarem alternativas para adequação da água aos novos padrões. Entre estas alternativas estava a utilização de uma camada intermediária de carvão ativado granular nos filtros lentos de areia. Esta tecnologia foi denominada de "carvão ativado granular (CAG) sanduíche" e consiste na substituição de uma camada intermediária de areia por uma camada de carvão ativado granular. A camada de areia sob a camada de CAG minimizava o risco de aumento de microrganismos no efluente de leitos de CAG somente (BAUER et al., 1996). O CAG tem sido responsável pela remoção de carbono orgânico dissolvido e microcontaminantes orgânicos, entre eles alguns agrotóxicos (PAGE et al., 1996; XIAOJIAN et al., 1991; JIANG e ADAMS, 2006; NIGEL et al., 2006; VELTEN et al., 2007; WHO, 2010).

Li et al. (2004) apresentaram a adsorção em CAG como possibilidade para remoção de ATZ, mas consideraram que a presença de matéria orgânica pode resultar na redução da eficiência de remoção. No entanto, tem sido reportado que a atividade biológica contribui para remoção deste herbicida em CAG (WANG \& ALBEN, 1998). As características da superfície do CAG fazem deste um meio mais favorável ao crescimento e formação de biofilme, quando comparado com o leito de areia. Esta vantagem tem ampliado o uso de CAG tornando-o uma alternativa interessante pelo aumento de sua vida útil sem regeneração.

Simpson (2008) denomina esta tecnologia de carvão ativado biológico e atribui ao biofilme formado a biodegradação de matéria orgânica dissolvida e de microcontaminantes, e a adsorção a retenção desubstancias não biodegradáveis nos poros do CAG. A biodegradação da matéria adsorvida serve para regenerar biologicamente o CAG e prolongar sua vida útil (aproximadamente dois a cinco anos). Ainda, segundo Simpson (2008), o efluente produzido apresenta menor conteúdo orgânico. Poucos estudos têm reportado a remoção de ATZ e seus metabólitos em leitos de CAG e para prever a remoção de microcontaminantes em leitos de CAG em águas naturais Scharf et al.(2010) sugerem a utilização de instalação piloto.

Considerando os problemas de saúde pública que podem ser causados pela presença de ATZ e seus produtos de degradação em águas de abastecimento,,a proposta do trabalho foi o estudo,em instalação piloto, do processo de filtração lenta em leito de areia e leito intermediário de CAGna remoção de ATZ e seus produtos de degradação DEA, DIA e DEHA.

\section{Material e métodos}

\section{Características da instalação piloto}

A instalação piloto era do tipo filtração em múltiplas etapas constituída de pré-filtro dinâmico seguido de pré-filtro vertical ascendente e unidades de filtração lenta de fluxo descendente, que foram operados com taxa constante igual a $4 \mathrm{~m}^{3} \cdot \mathrm{m}^{-2} \cdot \mathrm{dia}^{-1}$. Neste trabalho será apresentada somente a eficiência dos filtros lentos, sendo considerada como vazão afluente a esses filtros o efluente do pré-filtro vertical ascendente. Uma unidade de filtração lenta era constituída de leito filtrante único de areia (FLA), e outra,de leito de areia associado à camada intermediária de carvão ativado granular (FLACAG). A instalação foi alimentada com água bruta do Córrego Feijão (SP). O esquema das unidades de filtração lenta pode ser visualizado na Figura 1.

O leito filtrante do filtro de camada única de areia possuía espessura de 0,70 m, tamanho efetivo de 0,25 mm, coeficiente de uniformidade de 2,0 e granulometria entre 0,08 e 1,00 mm. O filtro com camada intermediária de carvão ativado se caracterizou pela substituição de $30 \mathrm{~cm}$ de areia por uma camada intermediária de CAG com granulometria entre 0,30-0,85 mm, coeficiente de desuniformidade $\leq 1,7$ e tamanho efetivo entre 0,4 e $0,45 \mathrm{~mm}$ e tempo de contato de 1,8 horas. Sobre a camada de CAG existia uma camada de areia de 0,1 m. O carvão utilizado foi proveniente da casca de coco fabricado por processo de ativação física, com volume de microporos igual a $0,315 \mathrm{~cm}^{3} \cdot \mathrm{g}^{-1}$ e área superficial específica de $567 \mathrm{~m}^{2} \cdot \mathrm{g}^{-1}$.

Para avaliar a capacidade da filtração lenta na remoção de ATZ e metabólitos foram realizadas duas Carreiras de filtração adicionando-se 


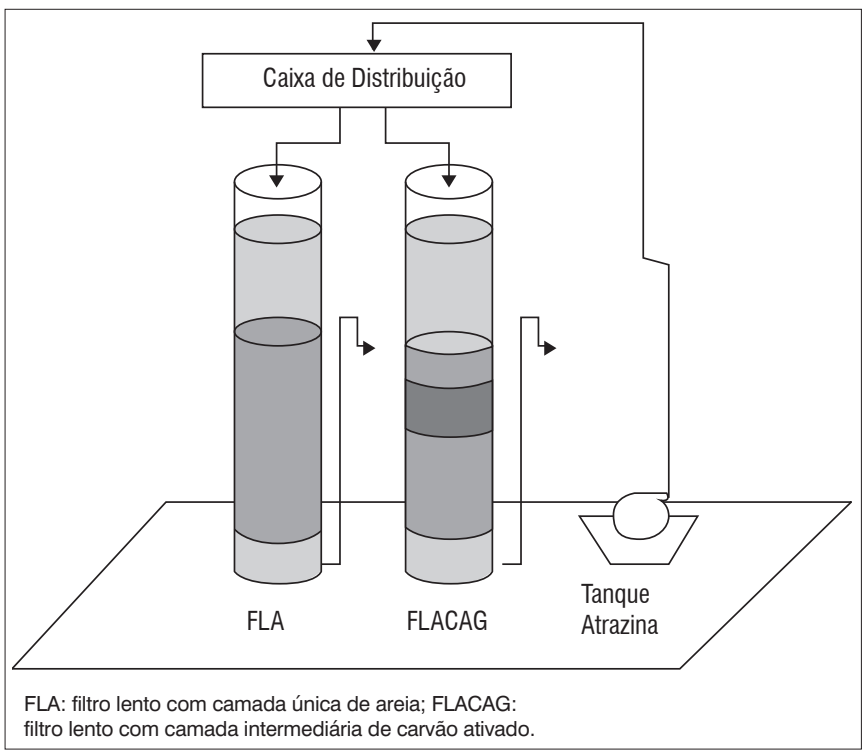

Figura 1 - Esquema da instalação piloto.

ATZ a montante dos filtros lentos. Na primeira Carreira (Carreira 1) foi avaliada a remoção de ATZ enquanto na segunda Carreira (Carreira 2) foram também avaliados a remoção dos subprodutos DEA, DIA e DEHA.

\section{Preparo da solução de atrazina}

Para adição de ATZ no sistema foi utilizada uma formulação comercial com concentração medida em laboratório de 280 g. $\mathrm{L}^{-1}$.

\section{Amostragem e parâmetros de controle}

Os pontos de amostragem foram: afluente aos filtros lentos (água proveniente do pré-filtro vertical ascendente), filtro lento com camada única de areia (FLA) e filtro lento com camada intermediária de carvão ativado (FLACAG).

Os parâmetros utilizados para monitorar e medir a qualidade da água com relação ao conteúdo orgânico foram: cor verdadeira (CV), absorvância-UV 254 nm, oxigênio consumido (OC) e carbono orgânico dissolvido não purgável (CODNP). As amostras utilizadas para medida de absorvância e CODNP foram filtradas em membrana de 0,45 mm ME-25 - SS. As amostras para medida de CV foram centrifugadas por 30 minutos a $3.500 \mathrm{rpm}$. A matéria particulada foi avaliada pela medida de turbidez e os microrganismos foram avaliados pela medida de coliformes totais e Escherichia colipelo método do substrato definido, utilizando-se enzima Colilert Idexx. A metodologia para análises e exames dos parâmetros mencionados está descrita em Standard Methods for the Examination of Water and Wastewater, APHA (1998).

\section{Identificação da atrazina e produtos de degradação}

Foram utilizados os reagentes analíticos, acetato de etila, hexano e metanol, com grau de pureza HPLC (99,9\%), da Mallinckrodt.
Os padrões de ATZ (grau de pureza de 98\%), desetilatrazina (pureza de 95,5\%), DIA (grau de pureza de 96,5\%) e desetilhidroxiatrina (grau de pureza de 97,5\%) foram obtidos do Labor Dr. Ehrenstorfer, Augsburg, Alemanha. As curvas analíticas foram traçadas pelo método da adição de padrão externo a partir de uma solução estoque. Para quantificação de ATZ e metabólitos nas amostras ambientais, foi realizada extração em fase sólida em cartucho LC-18 (octadecyl-500 mg/6 mL) da Supelco. Após o condicionamento das colunas, foram passados $500 \mathrm{~mL}$ de amostras através das colunas, por meio de um sistema de manifold a vácuo, com vazão de 8 a $10 \mathrm{~mL} \cdot \mathrm{min}^{-1}$. Após passar as amostras, as colunas foram secas no vácuo por 5 minutos e eluídas com volume de $5 \mathrm{~mL}$ de metanol, que foi reduzido para 1,0 mL com gás nitrogênio para posterior injeção.

\section{Cromatografia}

A determinação dos compostos foi realizada utilizando-se cromatógrafo líquido Shimadzu, modelo SPD-6A, equipado com detector UV-Vis LC-9 A (HPLC-UV); Coluna C $_{8}$ (Shim-pack). Todas as determinações foram realizadas no comprimento de onda de $230 \mathrm{~nm}$ (comprimento de onda de absorção da ATZ e dos subprodutos). A quantificação da ATZ e dos subprodutos foi realizada com as seguintes condições operacionais: mistura de metanol/água (70:30 v/v) como eluente; fluxo de $1 \mathrm{~mL} \cdot \mathrm{min}^{-1}$ com loop de $2 \mu \mathrm{L}$.

\section{Resultados e Discussão}

A Instalação Piloto foi alimentada com água bruta do manancial Córrego do Feijão.As características físicas, químicas e biológicas, durante o período das Carreiras de filtração, estão apresentadas na Tabela 1 .

Quando da realização da Carreira 1, o sistema já havia funcionado por 84 dias sem adição de ATZ. Esta condição permitiu avaliar a dinâmica de um sistema de tratamento recebendo água natural associado a sazonalidade da presença de agrotóxicos. A Carreira 1 teve duração de 94 dias, com volume de água tratada de $182 \mathrm{~m}^{3}$ com frequência de amostragem variável conforme Figura 2. A Carreira 2 foi iniciada após 263 dias de operação contínua do sistema de tratamento caracterizada

Tabela 1 - Características da água bruta durante as Carreiras de filtração.

\begin{tabular}{|c|c|c|}
\hline \multirow{2}{*}{ Características da água bruta } & \multicolumn{2}{|c|}{$\begin{array}{l}\text { Valores apresentados } \\
\text { (mínimo-máximo) }\end{array}$} \\
\hline & Carreira 1 & Carreira 2 \\
\hline Absorvância 254 nm (cm-1) & $0,038-0,092$ & $0,067-0,23$ \\
\hline CODNP (mg.L-1) & $0,35-1,9$ & $2,05-5,7$ \\
\hline Cor verdadeira $(\mathrm{uH})$ & $30-78$ & $64-125$ \\
\hline Oxigênio consumido (mg. $\mathrm{L}^{-1}$ ) & $0,95-3,4$ & $2,4-2,1$ \\
\hline Turbidez (uT) & $7-24$ & $22-181$ \\
\hline Coliformes totais (NMP/100 mL) & $1.541-6.867$ & $7.940-36.540$ \\
\hline Escherichia coli (NMP/100 mL) & $100-520$ & $100-2.180$ \\
\hline
\end{tabular}

CODNP: carbono orgânico dissolvido não purgável. 
por período de chuvas, justificando, assim, o menor tempo de duração e o intervalo de frequência de coleta de amostras foi duas vezes por semana. Entre as Carreiras 1 e 2 foi realizada uma Carreira intermediária, sem adição de ATZ com duração de 85 dias (dados não apresentados). $\mathrm{Na}$ Carreira 1, a ATZ foi adicionada no sétimo dia de operação e na Carreira 2 no segundo dia. No encerramento das carreiras era realizada a raspagem da camada superficial da areia.

\section{Atrazina e metabólitos}

Na Carreira 1, a ATZ foi adicionada em concentração variando de 0,20 a $287 \mu \mathrm{g} . \mathrm{L}^{-1}$, simulando a dinâmica de variação no meio ambiente. O FLA apresentou remoção de ATZ de 78 a 99\% quando a concentração no afluente variou de 0,18 a $15 \mu \mathrm{g} \cdot \mathrm{L}^{-1}$ nos primeiros 33 dias de operação. Esta remoção não foi observada nos dias e na Carreira subsequente. No decorrer da Carreira, a variação ficou entre os limites de nenhuma remoção e $87 \%$, não apresentando valores nominais inferiores a $2 \mu \mathrm{g} . \mathrm{L}^{-1}$ conforme estabelece a Portaria MS $\mathrm{n}^{\circ}$ 2.914/2011 (BRASIL, 2011). Resultados semelhantes, de baixa remoção de ATZ na filtração lenta em leito de areia, foram encontrados por Foster et al.(1991) e Woudneh et al.(1996).

No efluente do FLACAG, na Carreira 1, foi possível observar valores inferiores a $2 \mu \mathrm{g} . \mathrm{L}^{-1}$ quando a concentração no afluente foi inferior a $24 \mu \mathrm{g} . \mathrm{L}^{-1}$, até o $63^{\circ}$ dia de operação. Somente no final da carreira, no $82^{\circ}$ dia de operação, o valor para ATZ foi inferior a $2 \mu \mathrm{g} . \mathrm{L}^{-1}$ para concentração no afluente igual a $219 \mu \mathrm{g} \cdot \mathrm{L}^{-1}$. Resultados semelhantes para remoção de ATZ em leito de CAG foram encontrados por Bauer et al. (1996). Os valores para ATZ, para as Carreiras 1 e 2, podem ser visualizados na Figura 2.

$\mathrm{Na}$ Carreira 2, além da ATZ, foram avaliados os subprodutos DEA, DIA e DEHA. A concentração de ATZ adicionada ao afluente variou de 57 a $101 \mu \mathrm{g} . \mathrm{L}^{-1}$, e as concentrações dos subprodutos detectadas no afluente foram resultado da presença desses na solução de preparação da ATZ. É interessante ressaltar que no início desta Carreira o sistema já havia funcionado por 178 dias. No efluente do FLA ocorreu eficiência de remoção de ATZ entre 35 a 89\%, a partir do $11^{\circ}$ dia de operação, mas os valores nominais medidos foram superiores a $2 \mu \mathrm{g}$. $\mathrm{L}^{-1}$ não atendendo o padrão de potabilidade vigente no Brasil. No FLACAG pode-se observar valores nominais medidos sempre inferiores a $2 \mu \mathrm{g} . \mathrm{L}^{-1}$. O resultado, para remoção de ATZ das Carreira 1 e 2, indica que o processo de filtração lenta associado à camada intermediária de CAG pode ser proposto como tecnologia de remoção de ATZ para enquadramento ao padrão de potabilidade brasileiro. Estes resultados concordam com os resultados apresentados por Bauer et al. (1996) que estudou a remoção de quatro pesticidas, entre eles a ATZ, em filtro tipo "sanduiche" por 430 dias.

A legislação brasileira, Portaria MS no 2.914/2011 (BRASIL, 2011), não faz referência a concentração dos produtos de degradação da ATZ sendo que a discussão para estes será feita considerando o limite estabelecido pela legislação europeia para concentração de agrotóxicos de $0,1 \mu \mathrm{g} . \mathrm{L}^{-1}$ para valor individual e 0,5 $\mu \mathrm{g}$. $\mathrm{L}^{-1}$ para a somatória dos agrotóxicos na amostra.

A presença do metabólito DEA foi detectada no afluente e efluente dos filtros lentos em todas as amostras indicando a importância do monitoramento deste em sistemas de tratamento de água. A remoção nos filtros não foi significativa, sendo que $75 \%$ dos valores foram superiores a $0,1 \mu \mathrm{g} . \mathrm{L}^{-1}$ e não foi observado padrão de comportamento que indicasse essa tecnologia para remoção do metabólito DEA. Os dados estão apresentados na Figura 3.

O aparecimento dos metabólitos DIA e DEHA no afluente alternou entre presença e ausência (limite de detecção de 0,01 $\mu \mathrm{g} . \mathrm{L}^{-1}$ ). Considerando a mediana foi possível verificar ligeiro aumento da concentração no FLA. A presença destes no efluente dos filtros pode ser explicada pela baixa eficiência de remoção e pela formação destes como produtos de degradação da ATZ. Não foi observado comportamento distinto entre o FLA e

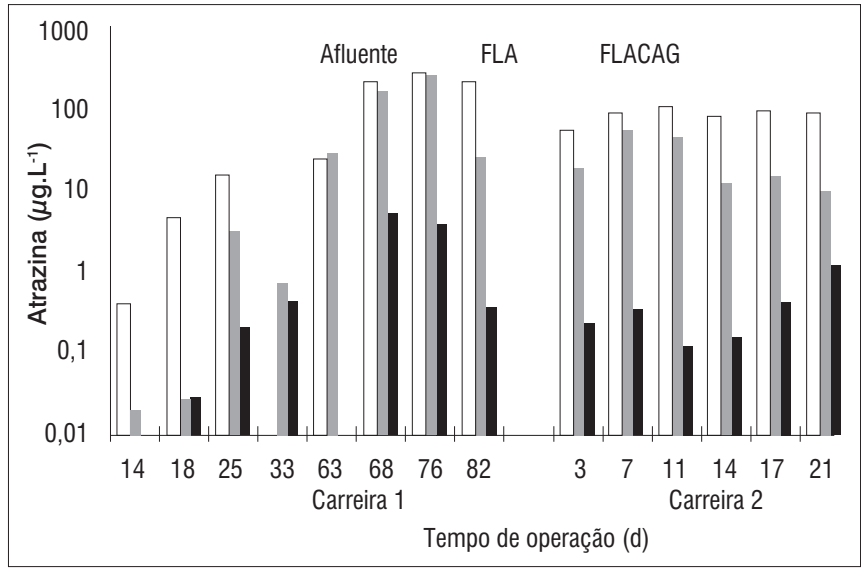

Figura 2 - Atrazina $\left(\mu \mathrm{g} . \mathrm{L}^{-1}\right)$ para afluente e efluente do leito filtrante único de areia e leito de areia associado à camada intermediária de carvão ativado granular - Carreiras 1 e 2.

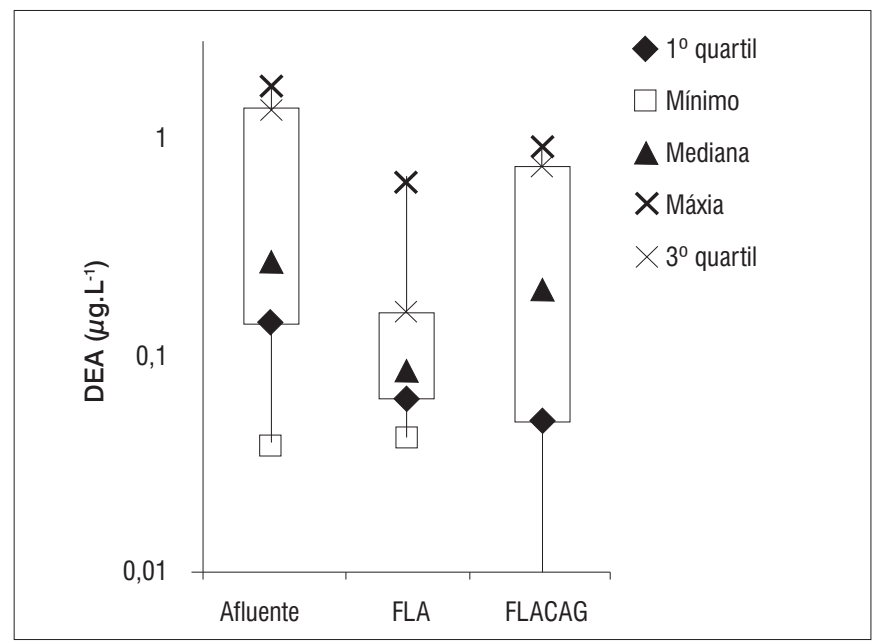

Figura 3 - Dietilatrazina $\left(\mu \mathrm{g} . \mathrm{L}^{-1}\right)$ para afluente e efluente do leito filtrante único de areia e leito de areia associado à camada intermediária de carvão ativado granular - Carreira 2 ( $n=6$, duas coletas semanais). 
FLACAG para o DIA, considerando o $3^{\circ}$ quartil com $75 \%$ valores superiores a $0,1 \mu \mathrm{g} . \mathrm{L}^{-1}$ e estes não se apresentaram como tecnologia aplicável na remoção deste. Os resultados podem ser visualizados na Figura 4.

Para o DEHA pode-se observar maior concentração no afluente quando comparado com o DIA e maior eficiência do FLACAG se considerar o $3^{\circ}$ quartil com 75 dos valores ( $n=6$ ) com concentração inferior a $0,02 \mu \mathrm{g} \cdot \mathrm{L}^{-1}$ quando a concentração no afluente for inferior a 2,4 $\mu \mathrm{g} \cdot \mathrm{L}^{-1}$ (Figura 5).

Pode-se considerar a importância de investigar a presença desses metabólitos em sistemas de abastecimento público considerando a frequência com que aparecem no afluente em concentração superior a 0,1 $\mu \mathrm{g} . \mathrm{L}^{-1}$ : DEA (em 100\% das amostras) >DEHA e DIA (em $50 \%$ das amostras). Pode-se observar também baixa remoção, no conteúdo do DEA, DIA e DEHA para valores inferiores a 0,1 $\mu \mathrm{g} . \mathrm{L}^{-1}$, evidenciando a limitação do processo de filtração lenta em leito de areia somente e associado à camada intermediária de CAG. A baixa capacidade de remoção dos metabólitos pode estar associada à maior solubilidade destes quando comparados com a da ATZ, obedecendo a lei empírica denominada regra de Lundelius, que preconiza que de modo geral, a extensão da adsorção de um soluto é inversamente proporcional a sua solubilidade no solvente do qual ocorre a adsorção. Resultados semelhantes foram encontrados por Adams e Watson (1996) para DEA e DIA em ensaios de isotermas.

Considerando que as s-triazinas, ATZ, DEA, DIA são conhecidos como disruptores endócrinos e que a somatória das concentrações destesforam superior a $2 \mu \mathrm{g} . \mathrm{L}^{-1}$, pode-se inferir que existe risco a saúde.Vale lembrar que para a comunidade europeia o limite é 0,5 $\mu \mathrm{g} \cdot \mathrm{L}^{-1}$ para somatória de agrotóxicos na amostra. Na Portaria MS no 2.914/2011 (BRASIL, 2011) não existe padrão de referência para estes metabólitos. A importância do estudo desses metabólitos pode ser corroborada pela atual reavaliação e discussão pela EPA da inserção em seu padrão de potabilidade alguns metabólitos da ATZ (USEPA, 2009; WHO, 2010). O processo de filtração lenta com camada intermediária de CAG foi eficiente na remoção de ATZ para concentração no afluente inferior a $147 \mu \mathrm{g} . \mathrm{L}^{-1}$, considerando o limite máximo permitido de $2 \mu \mathrm{g} . \mathrm{L}^{-1}$ no padrão de potabilidade brasileiro e, não foi observada remoção dos metabólitos indicando que o processo não foi eficiente na remoção destes quando a concentração afluente foi inferior a $2 \mu \mathrm{g} \cdot \mathrm{L}^{-1}$.

Para o controle de todo o processo de filtração lenta outros parâmetros foram monitorados, entre eles os apresentados na Tabela 1. Dentre esses parâmetros será apresentado o resultado do conteúdo orgânico avaliado pela medida de absorvância no comprimento de onda de $254 \mathrm{~nm}$. O FLACAG apresentou efluente mais estável quando comparado com o FLA, concordando com Page et al. (1996). O resultado pode ser visualizado na Figura 6. Esta medida serviu como indicação da interferencia da matéria orgânica no processo de remoção da ATZ em CAG. Na Carreira 2 ocorreu uma elevação considerável na medida da absorvância e não foi observado comprometimento na remoção de ATZ com produção de água filtrada com valores inferiores a $2 \mu \mathrm{g} . \mathrm{L}^{-1}$ para concentração no afluente inferior a $147 \mu \mathrm{g} \cdot \mathrm{L}^{-1}$ e tempo total de

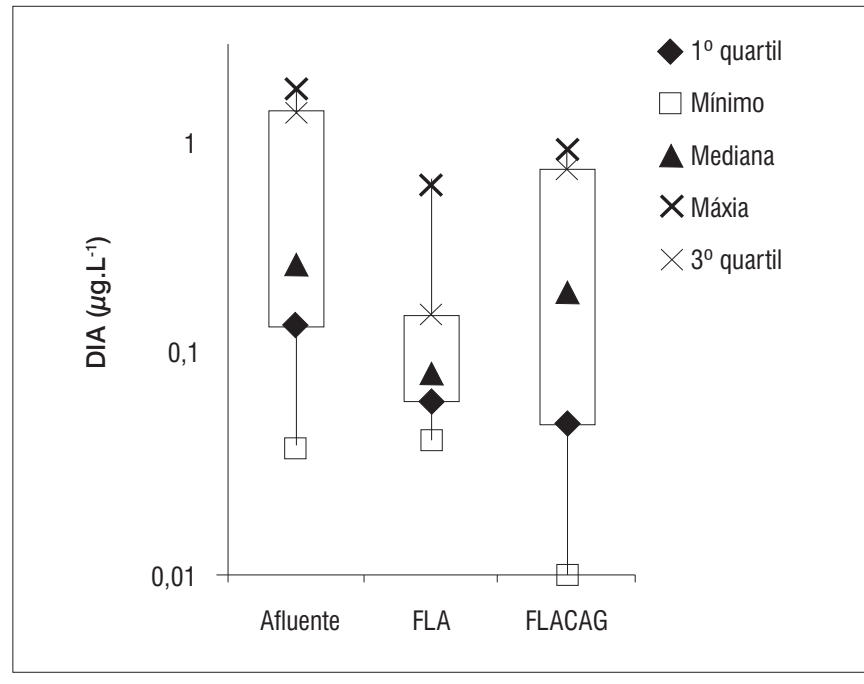

Figura 4 - Deisopropilatrazina ( $\left.\mu \mathrm{g} . \mathrm{L}^{-1}\right)$ para afluente e efluente do leito filtrante único de areia e leito de areia associado à camada intermediária de carvão ativado granular- Carreira 2 ( $n=6$, duas coletas semanais).

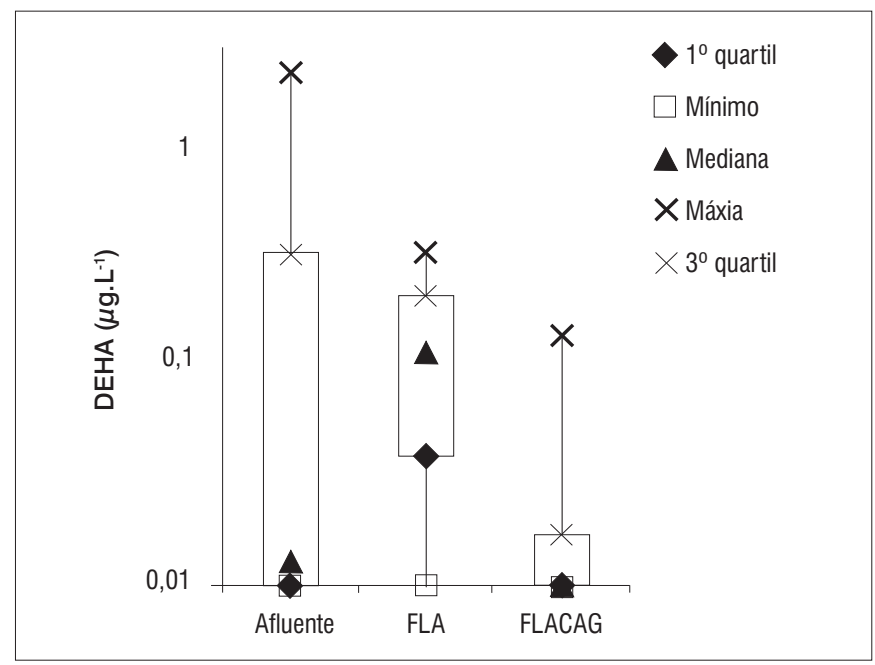

Figura 5 - Dietilhidroxiatrazina $\left(\mu \mathrm{g} . \mathrm{L}^{-1}\right)$ para afluente e efluente do leito filtrante único de areia e leito de areia associado à camada intermediária de carvão ativado granular- Carreira 2 ( $n=6$, duas coletas semanais).

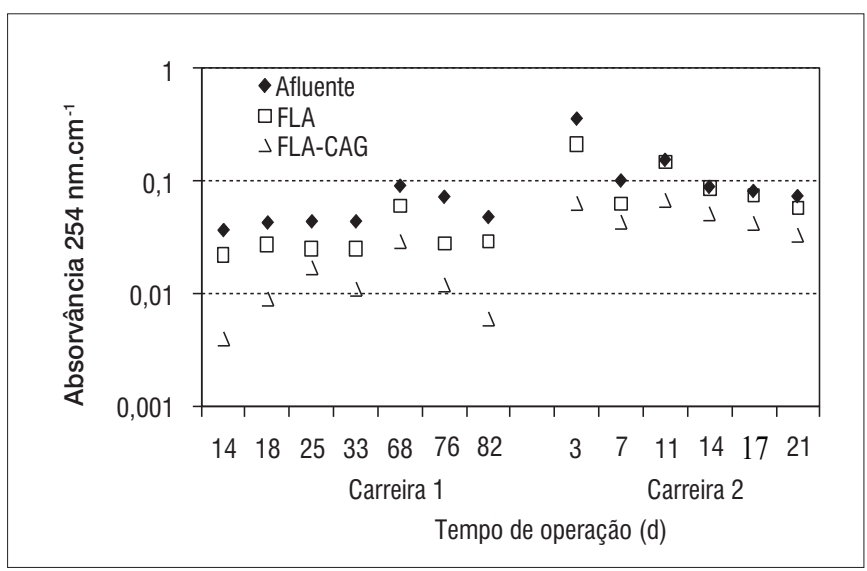

Figura 6 - Absorvância $254 \mathrm{~nm} \cdot \mathrm{cm}^{-1}$ para afluente e efluente do leito filtrante único de areia e leito de areia associado à camada intermediária de carvão ativado granular - Carreiras 1 e 2. 
operação do filtro foi de 284 dias. Resultados semelhantes para matéria orgânica dissolvida e bifenilas policloradas foram encontrados por Page et al.(1996) e Ghosh et al. (1999), respectivamente.

\section{Conclusões}

Com o aumento das evidências dos potenciais efeitos da ATZ e seus metabólitos clorados como disruptores endócrinos, o estudo da presença e remoção desses em processos de tratamento de água para abastecimento público torna-se de grande importância. Considerando-se a tecnologia de filtração lenta associada à camada intermediária de CAG como tecnologia para remoção da ATZ e metabólitos foi possível verificar que:
- O filtro lento com camada única de areia não foi eficiente na remoção de ATZ, DEA, DIA e DEHA;

- O filtro lento de areia associado à camada intermediária de CAG apresentou efluente com valores de ATZ inferiores a $2 \mu \mathrm{g} . \mathrm{L}^{-1}$, quando no afluente os valores foram inferiores a $147 \mu \mathrm{g} . \mathrm{L}^{-1}$, podendo ser avaliada como tecnologia para remoção de ATZ;

- O subproduto DEA foi detectado no afluente e efluente dos filtros lentos em todas as amostras, sendo recomendável a medida deste metabólito associadoao controle da ATZ em sistemas de abastecimento de água;

- O filtro lento de areia associado à camada intermediária de CAG não se apresentou como tecnologia viável para redução no conteúdodos metabólitos DEA, DIA e DEHA.

\section{Referências}

ABATE, G.; MASINI, J.C. (2005) Sorption of atrazine, propazine, deethylatrazine, deisopropylatrazine and hudroxyatrazine onto organovermiculite. Journal of the Brazilian Chemical Society, v. 16, n. 5, p. $936-943$

ADAMS, C.D.; WATSON, T.L. (1996) Treatability of s-triazine herbicide metabolites using powered activated carbon. Journal of Environmental Engineering, v. 122, n. 4, p. 327-330

AMERICAN PUBLIC HEALTH ASSOCIATION (APHA). (1998) Standard methods for the examination of water and wastewater. 20th ed. Washington D.C: APHA.

BAUER, M.J.; COLBOURNE, J.S.; FOSTER, D.M.; GOODMAN, N.V.; RACHWAL, A.J. (1996) GAC Enhanced Slow Sand Filtration (GAC Sandwich). In: GRAHAM, N.; COLLINS, R. Advances in slow sand an alternative biological filtration. Chichester: John Wiley \& Sons, p. 223-232.

BIRADAR, D.P.; RAYBURN, A.L. (1995) Chromosomal damage induced by herbicide contamination at concentrations observed in public water supplies. Journal of Environmental Quality, v. 24, n. 6, p. 1222-1225.

BORTOLUZZI, E.C.; RHEINHEIMER, D.S.; GONÇALVES, C.S.; PELLEGRINI, J.B.R.; ZANELLA, R.; COPETTI, A.C.C. (2006) Contaminação de águas superficiais por agrotóxicos em função do uso do solo numa microbacia hidrográfica de Agudo, RS. Revista Brasileira de Engenharia Agrícola Ambiental, v. 10, n. 4, p. 881-887.

BRASIL. Portaria n².914 de 12 dezembro de 2011. Padrão de potabilidade. Ministério da Saúde.

CEREJEIRA, M.J.; VIANA, P.; BATISTA, S.; PEREIRA, T.; SILVA, E.; VALÉRIO, M.J.; SILVA, A.; FERREIRA, M.; SILVA-FERNANDES, A.M.. (2003) Pesticides in Portuguese surface and ground waters. Water Research,v. 37, n. 5, p. 1055-1063.

FOSTER, D.M.; RACHWAL, A.J.; WHITE, S.L. (1991) New treatment processes for pesticides and chlorinated organics control in drinking water. Water and Environmental Journal, v. 5, n. 4, p. 466-476.
FRIEDMANN, A.S. (2002) Atrazine inhibition of testosterone production in rat males following peripubertal exposure. Reproductive Toxicology, v. 16, n. 3, p. $275-279$

GIBSON, G.; KOIFMAN, S. (2008) Consumo de agrotóxicos e distribuição temporal da proporção de nascimentos masculinos no Estado do Paraná, Brasil. Revista Panamericana de Salude Pública, v. 24, n. 4. p. 240-247

GHOSH, U.; WEBER, A.S.; JENSEN, J.N.; SMITH, J.R. (1999) Granular activated carbon and biological activated carbon treatment or dissolved and sorbed polychlorinated biphenyls. Water Environment Research, v. 71, n. 2 , p. $232-240$.

GRAYMORE, M.; STAGNITTI, F.; ALLINSON, G. (2001) Impacts of atrazine in aquatic ecosystems. Environment International, v. 26. n. 7-8, p. 483-495.

HAYES, T.B.; STUART, A.A.; MENDOZA, M.; COLLINS, A.; NORIEGA, N.; JOHNSTON, G.; LIU, R.; KPODZO, D. (2006) Characterization of atrazineinduced gonadal malformations in African clawed frogs (Xenopus laevis) and comparisons with effects of an androgen antagonist (cyproterone acetate) and exogenous estrogen (17 $\beta$-Estradiol): support for the demasculinization/feminization hypothesis castration in male African clawed frogs. Environmental Health Perspectives, v. 114, Suppl. 1, p. 134-141.

JIANG, H. \& ADAMS, C. (2006) Treatability of chloro-s-triazines by conventional drinking water treatment technologies. Water Research, v. 40, n. 8, p. 1657-1667.

LAABS, V.; AMELUNG, W.; PINTO, A.; WANTZEN, M.; SILVA, C.; ZECH, W. (2002). Pesticides in surface water, sediment, and rainfall of the northeastern Pantanal basin, Brazil. Journal of Environmental Quality, v. 31, n. 5, p. 1636-1648.

LI, Q. MARIÑAS, B.J.; SNOEYINK, V.L.; CAMPOS, C. (2004) Pore blockage effect on atrazine adsorption in powered activated carbon/ membrane system. I: Model development. Journal of Environmental Engineering, v. 130, n.11, p.1242-1252. 
MBWETTE, T.S.A.; STEITIEH, M.A.R.; GRAHAM, N.J.D. (1990) Performance of fabric protected slow sand filtration treating lowland surface water. Water and Environmental Journal, v. 4, n.1, p. 51-61.

MILTNER, R.J.; BAKER, D.B.; SPETH, T.F; FRONK, C.A. (1989) Treatment of seasonal pesticides in surface water. Journal American Water Works Association, v. 81, n. 1, p. 43-52.

NIGEL, R.G.; GRAHAM, N.J.D.; COLLINS, M.R. (2006) Recent progress in slow sand and alternative biofiltration processes. IWA, London.

OH, S.M; SHIM, S.H; CHUNG, K.H. (2003) Antiestrogenic action of atrazine and its major metabolites in vitro. Journal of Health Science, v. 49, n. 1, p. 65-71.

PAGE, T.G.; COLLINS, M.R.; BAUER, M.J.; RACHWAL, A.J. (1996) NOM removals by "GAC sandwich" modification to slow sand filtration. In: GRAHAM, N.; COLLINS, R. Advances in slow sand an alternative biological filtration. Chichester: John Wiley \& Sons, p. 223-232.

PROGRAMA DE PESQUISA EM SANEAMENTO BÁSICO (PROSAB). (1999) Tratamento de águas de abastecimento por filtração em múltiplas etapas. ABES. Rio de Janeiro.

SCHARF, R.F.; JOHNSTON, R.W.; SEMMENS, M.J.; HOZALSKI, R.M. (2010) Comparison of batch sorption testes, pilot studies, and modeling for estimating GAC bed life. Water Research, v. 44, p. 769-780.

SIMPSON, D.R. (2008) Biofilm processes in biologically active carbon water purification. Water Research, v. 42, n. 12, p. 2839-2848.

SWAN, S.H.; KRUSE, R.L.; LIU, F.; BARR, D.B.; DROBNIS, E.A.; REDMON, J.B.; WANG, C.; BRAZIL, C.; OVERSTRET, J.W.; STUDY FOR FUTURE FAMILIES RESEARCH GROUP. (2003) Semen quality in relation to biomarkers of pesticide exposure. Environmental Health Perspectives, v. 111, n. 12, p. 1478-1484.

THUY, P.T.; MOONS, K.; VAN DIJK, J.C.; ANH, N.V.; VAN DER BRUGGEN, B. (2008) To what extent are pesticides removed from surface water during coagulation-flocculation? Water and Environment Journal, v. 22, n. 3, p. 217-223.

UETA, J; SHUHAMA, I.K.; CERDEIRA, A.L. (2001). Biodegradação de herbicidas e biorremediação: microrganismos degradadores de atrazina provenientes de solos da Região do Aqüífero Guarani. [Internet] Revista Plantio Direto. 1 fev. 2001. Disponível em: http://plantiodireto. com.br/?body=cont_int\&id=81. Acesso em: 27 nov. 2012.

U.S. ENVIRONMENTAL PROTECTION AGENCY (USEPA). (2009) Final list of chemicals for initial tier 1 screening: overview of the April 2009. Washington, DC, United States Enrironmental Protection Agency. Disponível em: http://www.epa.gov/endo/pubs/prioritysetting/finallist. htm. Acesso em: 3 dez. 2012.

VELTEN, S.; HAMMES, F.; BOLLER, M.; EGLI, T. (2007) Rapid and direct estimation of active biomass on granular activate carbon through adenosine tri-phosphate (ATP) determination. Water Research, v. 41, n. 9. p. 1973-1983.

XIAOJIAN, Z.; ZHANSHENG, W.; XIASHENG, GU. (1991) Simple combination or biodegradation and carbon adsorption - the mechanism of the biological activated carbon process. Water Research, v. 25, n. 2, p. 165-172.

WANG, G.S.; ALBEN, K.T. (1998) Effects of preadsorbed natural organic matter on granular activated carbon adsorption of atrazine. Science Total Environment, v. 224, n. 1-3, p. 221-226.

WORLD HEALTH ORGANIZATION (WHO). (2010) Atrazine and its metabolites in drinking-water-Background document for development of WHO Guidelines for Drinking Water. Disponivel em: http://www.who.int/ entity/water_sanitation_health/dwq/chemicals/Fourth_Edition_Atrazine_ Document_Draft.doc. Acesso em: 3 dez. 2012.

WOUDNEH, B. J.; LLOYD, B.J.; STEVENSON, D. (1996) Removal of herbicides by biological filter. In: GRAHAM, N.; COLLINS, R. Advances in slow sand and alternative biological filtration. Chichester: John Wiley \& Sons, p.211-221. 